\title{
Consensus-based formation control of networked nonholonomic vehicles with delayed communications
}

\author{
Mohamed Maghenem Antonio Loría Emmanuel Nuño Elena Panteley
}

\begin{abstract}
For a network of nonholonomic vehicles communicating according to an undirected connected graph, a consensus-based formation control problem is solved via a smooth time-varying, proportionalderivative, $\delta$-persistently-exciting, controller. It is assumed that the communication between agents is affected by time-varying, non-differentiable, communication delays and uniform global asymptotic stability is demonstrated. This goes beyond the more-often encountered property of nonuniform convergence and, what is more, for the first time in the literature, a strict Lyapunov-Krasovskiĭ functional is provided.
\end{abstract}

Index Terms-Formation control, persistency of excitation, Lyapunov design, nonholonomic systems

\section{INTRODUCTION}

For a group of (first or second order) integrators the problem of consensus, which consists in the state variables of all agents converging to a common value, is well studied and solved under many different scenarios [1]. For instance, for simple integrators interconnected over an undirected, static and connected graph, the consensus equilibrium corresponds to the (weighted) average to the initial conditions. However, the problem is more complex if one considers the agents' dynamics [2], [3], network constraints, such as communication delays [4], [5], or nonholonomic constraints that restrict the systems' motion [6].

For autonomous nonholonomic vehicles, which can obviously not converge to a common position, the objective may be formulated as the requirement that the vehicles converge to specific positions to form a geometric pattern anywhere on the plane. While the formation is predetermined by defining a position vector originating at the center of the formation, the said center is not imposed a priori, but it results from the initial positions, the network's topology and the dynamic behavior of the agents. We refer to this goal as consensus-based formation control.

As a set-point stabilization problem, consensus-based formation control for nonholonomic systems is not solvable via smooth timeinvariant feedback. Akin to [7], [8] for the case of one robot, in [6] necessary and sufficient conditions for consensus of nonholonomic vehicles are established.

From a control-theory viewpoint, it is relevant to make a distinction according to the model that is used as well as the state variables that are regulated. In terms of modelling, in many works a kinematicsonly (hence simplified) model is used — see e.g., [6], [9], [10], and [11]. In this note we employ a force-controlled (hence full) model — $f$. [12], [13]. Concerning the regulated variables, in some works

M. Maghenem is with the University of California at Santa Cruz. Email: mmaghene@ucsc.edu. E. Nuño is with the Dept. of Computer Science CUCEI, at University of Guadalajara, Guadalajara, Mexico. E-mails: emmanuel.nuno@cucei.udg.mx. A. Loría and E. Panteley are with the CNRS. LSS-CentraleSupelec, 3 Rue Joliot Curie, 91192, France. E-mails: loria(panteley)@1ss.supelec.fr. E. Panteley is also with ITMO University, Kronverkskiy av. 49, Saint Petersburg, 197101, Russia.

This article was supported by: Dept. STITS of University of Paris Saclay, by the Government of Russian Federation (grant 08-08), by the French National Research Agency via the project HANDY (grant number ANR-18-CE400010), by ECOS-Nord contract no. M14M02, by the Mexican CONACyT Basic Scientific Research grant CB-282807, and by CEFIPRA under the grant number 6001-A the orientation of the vehicles is completely disregarded [6], so formation control applies only to the Cartesian positions of a point on the plane. In this note, as in [14], we address the problem of partial consensus which pertains to the case in which all the agents are required to converge to a formation around an unknown center and the orientation of each vehicle is required to be stabilized to a desired given reference. In [15], [10], [17], [16] the full consensus problem is addressed; that is, it is also required for the robots to acquire a common, non- $a$ priori given, orientation. Other problems' classifications may be made rather in terms of the communications topology that is employed, as well as on the nature of available measurements, regardless of the model used [18].

Measurements may come from global-positioning systems [10], from proximity sensors [11], or both [9]. The first yield usually reliable measurements with respect to a fixed frame and may be used to achieve formation consensus from initially far-away positions; the latter deliver relative distances and orientations and are useful to maintain the formation while tracking a trajectory. In this note it is assumed that global-positioning measurements are available and that each robot communicates its position and orientation to a set of neighbors through a wireless network. Consequently, the information that each agent receives is affected by a delay.

Consensus of nonholonomic vehicles under delayed measurements is addressed, for instance, in [9], [11] and [19]. In [9] the delays are assumed to be constant, in [11] the delays are assumed to be constant and equal for all agents, and in [19], they are assumed to be differentiable with bounded derivatives (up to the fourth). In this note, we relax these hypotheses by assuming that the delays are timevarying and non-differentiable, and bounded. Such relaxations are clearly motivated by realistic scenarios such as in the case of package dropouts, intermittent communications, blackouts, etc. [4], [20].

The main contribution, however, is of a more theoretical nature. We prove uniform global asymptotic stability for the closed-loop system, which is a much stronger property than mere (non-uniform) convergence, as most-often encountered in the literature (including most of the previous references). Establishing uniform global asymptotic stability is crucial for time-varying nonlinear systems because, unlike convergence, it guarantees robustness of the system with respect to bounded disturbances. Furthermore, for the first time in the literature, we provide a strict Lyapunov-Krasovskiı functional. A significant use of the latter is in incorporating further restrictions in the control problem, such as obstacle avoidance, but this is not addressed here.

This note is a byproduct of [21] and it builds upon the previous articles [14], [19], and [22]. In [19] only convergence is demonstrated under more restrictive conditions on the time delays. In [14] and [22] uniform global asymptotic stability is established, but in the absence of delays. We also emphasize that our controller, which is inspired from that in [19], is smooth time-varying and of the $\delta$-persistentlyexciting class [23]. The crafting of the strict Lyapunov-Krasovskiı functional relies on the powerful Mazenc construction [24], [25].

In the next section we describe the networked systems' model and we formulate the problem at hand. The main result is presented in Section III and some simulation results are provided in Section IV. Concluding remarks are presented in Section V. 


\section{MODEL AND PROBLEM FORMULATION}

Let us consider a swarm of $N$ autonomous vehicles modeled as unicycles, hence, with kinematics

$$
\begin{aligned}
\dot{x}_{i} & =v_{i} \cos \left(\theta_{i}\right) \\
\dot{y}_{i} & =v_{i} \sin \left(\theta_{i}\right) \\
\dot{\theta}_{i} & =\omega_{i}, \quad i \leq N
\end{aligned}
$$

and dynamics

$$
\begin{aligned}
\dot{v}_{i} & =u_{v i} \\
\dot{\omega}_{i} & =u_{\omega i} .
\end{aligned}
$$

The variables $x_{i}$ and $y_{i}$ denote Cartesian coordinates of a fixed point on the vehicle relative to a fixed frame, $\theta_{i}$ denotes its orientation with respect to the horizontal axis, and $v_{i}$ and $\omega_{i}$ denote the forward and angular velocities respectively. The pair $\left(u_{v i}, u_{\omega i}\right)$ corresponds to the control inputs, which may depend on the current vehicle's local coordinates and time, but also on the coordinates of its neighbors.

Remark 1: We emphasize that, in contrast with a greater body of literature (e.g., [6], [9], [15], and [11]) in which the dynamics (2) is neglected, we assume here that the nonholonomic vehicle is torque controlled. From a physical viewpoint, there exists a direct relation between the wheels' input torque and the controls $\left(u_{v i}, u_{\omega i}\right)$. For simplicity, however, we model the dynamics using an integrator. There is little loss of generality in such simplification since, typically, Equations (2) correspond to those of a fully-actuated (hence feedback linearizable) Lagrangian system [12], [13].

It is assumed that the $i$ th vehicle communicates its positions $\left(x_{i}, y_{i}\right)$ and its orientation $\theta_{i}$ to a group of neighbors over a network; the topology of which is assumed to be defined by a connected, undirected, and static graph. The connectivity of the latter is defined by the Laplacian $L:=\left[\ell_{i j}\right] \in \mathbb{R}^{N \times N}$, where

$$
\ell_{i j}=\left\{\begin{array}{cl}
\sum_{j \in \mathcal{N}_{i}} a_{i j} & \text { if } i=j \\
-a_{i j} & \text { if otherwise, }
\end{array}\right.
$$

$a_{i j}>0$ if the $i$ th and $j$ th vehicles communicate with each other, and $a_{i j}=0$ otherwise.

Remark 2: By construction, $L$ has a zero row sum, i.e., $L \mathbf{1}_{N}=0$, where $\mathbf{1}_{N} \in \mathbb{R}^{N}$ corresponds to the vector $[1 \cdots 1]^{\top}$. Moreover, since the interconnection graph is connected, undirected and static, $L$ is symmetric, it has a single zero-eigenvalue, and the rest of the spectrum of $L$ is positive. Thus, $\operatorname{rank}(L)=N-1$.

For a group of $N$ vehicles, we formulate the formation control problem, consisting in making the vehicles take positions according to a geometric pattern centered at a position on the plane not known a priori, as a consensus problem. To that end, let $z_{c}:=\left[x_{c}, y_{c}\right]^{\top}$ denote the unknown coordinates of the said center and, for each $i \leq N$, let $\delta_{i}:=\left[\delta_{x i}, \delta_{y i}\right]^{\top}$ denote a vector compatible with physical constraints. Then, defining

$z_{i}:=\left[\begin{array}{c}x_{i}-\delta_{x_{i}} \\ y_{i}-\delta_{y_{i}}\end{array}\right], \quad z:=\left[z_{1}^{\top} \cdots z_{N}^{\top}\right]^{\top}, \quad v:=\left[v_{1} \cdots v_{N}\right]^{\top} \in \mathbb{R}^{N}$,

one may set as control objective that

$$
\lim _{t \rightarrow \infty} z(t)=\mathbf{1}_{N} \otimes z_{c}, \quad \lim _{t \rightarrow \infty} v(t)=0
$$

A more complete formulation, however, is the so-called called partial consensus [14]. In this reference it is required that, in addition to (5), the orientation angles converge to respective given constant references $\theta_{d i}$, that is,

$$
\lim _{t \rightarrow \infty} \theta(t)=\theta_{d} \quad \lim _{t \rightarrow \infty} \omega(t)=0
$$

where $\theta:=\left[\theta_{1} \cdots \theta_{N}\right]^{\top} \in \mathbb{R}^{N}$, idem $\theta_{d}$ and $\omega$.

For nonlinear non-autonomous systems, however, the properties (5) and (6) are fairly weak since they do not include neither stability nor uniformity and, hence, offer no guarantee of robustness with respect to external disturbances. In contrast to this, uniform (global) asymptotic stability guarantees that the system is totally stable [26]. For this reason in this note we solve the more challenging problem of rendering the consensus set

$$
\mathcal{S}_{p c}:=\left\{z=\mathbf{1}_{N} \otimes z_{c}, \quad \theta=\theta_{d}, \quad v=\omega=0\right\}
$$

uniformly globally asymptotically stable, for the system

$$
\begin{aligned}
\dot{z} & =\Phi(\theta) v \\
\dot{v} & =u_{v} \\
\dot{\theta} & =\omega \\
\dot{\omega} & =u_{\omega},
\end{aligned}
$$

where $\Phi(\theta)=\operatorname{diag}\left[\phi\left(\theta_{i}\right)\right], \phi\left(\theta_{i}\right):=\left[\cos \left(\theta_{i}\right) \sin \left(\theta_{i}\right)\right]^{\top}, u_{v}:=$ $\left[u_{v 1} \cdots u_{v N}\right]^{\top}$, and $u_{\omega}:=\left[u_{\omega 1} \cdots u_{\omega N}\right]^{\top}$.

Remark 3: Uniform global asymptotic stability of $\mathcal{S}_{p c}$ implies (5) and (6), but not the opposite.

Now, for the purpose of analysis via Lyapunov's direct method, we redefine the consensus problem for (7) as one of stabilization of an equilibrium, as opposed to that of a set. To that end, let

$$
\begin{aligned}
e_{i} & =\phi\left(\theta_{i}\right)^{\top} \sum_{j \in \mathcal{N}_{i}} a_{i j}\left(z_{i}-z_{j}\right), \\
s_{i} & =\phi\left(\theta_{i}\right)^{\perp \top} \sum_{j \in \mathcal{N}_{i}} a_{i j}\left(z_{i}-z_{j}\right),
\end{aligned}
$$

where $\phi\left(\theta_{i}\right)^{\perp}=\left[\sin \left(\theta_{i}\right)-\cos \left(\theta_{i}\right)\right]^{\top}$ or, in compact form,

$$
e=\Phi(\theta)^{\top} \mathcal{L} z, \quad s=\Phi(\theta)^{\perp \top} \mathcal{L} z,
$$

where $\mathcal{L}:=L \otimes I_{2}, \Phi(\theta)^{\perp}=\operatorname{diag}\left[\phi\left(\theta_{i}\right)^{\perp}\right], e:=\left[e_{1}^{\top} \cdots e_{N}^{\top}\right]^{\top}$, and $s:=\left[s_{1}^{\top} \cdots s_{N}^{\top}\right]^{\top}$. The advantage of this change of variable is that, since the matrix $\left[\Phi(\theta) \Phi(\theta)^{\perp}\right]$ is invertible for all $\theta \in \mathbb{R}^{N}$ and the communication graph is connected,

$$
\mathcal{L} z=0 \Leftrightarrow(e, s)=(0,0) .
$$

Thus, defining $\tilde{\theta}:=\theta-\theta_{d}$, uniform global asymptotic stability of the set $\mathcal{S}_{p c}$ is equivalent to that of the equilibrium point $(e, s, \tilde{\theta}, v, \omega)=(0,0,0,0,0)$. This is established in the next section.

\section{MAIN RESUlts}

\section{A. Control design and main statement}

We use a certainty-equivalent version of the $\delta$-persistently-exciting controller proposed in [14], in the absence of delays; this yields

$$
\begin{aligned}
& u_{v}=-K_{d t} v-K_{p t} e_{d}, \\
& u_{\omega}=-K_{d \theta} \omega-K_{p \theta} \tilde{\theta}-p(t) \kappa\left(s_{d}, e_{d}\right),
\end{aligned}
$$

where $K_{d t}, K_{p t}, K_{d \theta}$, and $K_{p \theta}$ are diagonal positive definite matrices, $\tilde{\theta}:=\theta-\theta_{d}, \kappa: \mathbb{R}^{2 N} \rightarrow \mathbb{R}^{N}$ is defined as

$$
\kappa\left(s_{d}, e_{d}\right):=\frac{1}{2}\left[s_{d 1}^{2}+e_{d 1}^{2}, \cdots, s_{d N}^{2}+e_{d N}^{2}\right]^{\top},
$$

and $e_{d}:=\left[e_{d 1}, \cdots e_{d N}\right]^{\top}$ and $s_{d}:=\left[s_{d 1}, \cdots s_{d N}\right]^{\top}$ are the measured errors affected by bounded delays $t \mapsto T_{i j}(t)$, that is, for each $i \leq N$,

$$
\begin{aligned}
e_{d i} & =\phi\left(\theta_{i}\right)^{\top} \sum_{j \in \mathcal{N}_{i}} a_{i j}\left(z_{i}(t)-z_{j}\left(t-T_{i j}(t)\right)\right), \\
s_{d i} & =\phi\left(\theta_{i}\right)^{\perp \top} \sum_{j \in \mathcal{N}_{i}} a_{i j}\left(z_{i}(t)-z_{j}\left(t-T_{i j}(t)\right)\right) .
\end{aligned}
$$


The term $p(t) \kappa\left(s_{d}, e_{d}\right)$ is an essential component of the control law (13). It is assumed that this term is $\delta$-persistently-exciting [27] that is, for any $\delta>0$ there exist $T_{\delta}$ and $\mu_{\delta}>0$ such that

$$
\left|\left[\begin{array}{l}
e_{d} \\
s_{d}
\end{array}\right]\right| \geq \delta \Longrightarrow \int_{t}^{t+T_{\delta}} \kappa\left(e_{d}, s_{d}\right)|p(\tau)| d \tau \geq \mu_{\delta} \quad \forall t \geq 0
$$

where $|\cdot|$ denotes the Euclidean norm. In turn, for the condition (16) to hold, it is necessary to impose that the function $p: \mathbb{R}_{\geq 0} \rightarrow \mathbb{R}$ be persistently exciting in the usual sense, that is, that there exist $T$ and $\mu>0$ such that

$$
\int_{t}^{t+T}|p(\tau)| d \tau \geq \mu \quad \forall t \geq 0 .
$$

Furthermore, we impose the following conditions on the function $t \mapsto p(t)$ and its derivatives:

Assumption 1: There exists $b_{p}>0$ such that

$$
\max \left\{|p|_{\infty},|\dot{p}|_{\infty},|\ddot{p}|_{\infty},\left|p^{(3)}\right|_{\infty}\right\} \leq b_{p},
$$

where $|p|_{\infty}:=\sup _{t>0}|p(t)|$ and, moreover, $\dot{p}(t)$ is persistently exciting with excitation parameters $\left(T_{p}, \mu_{p}\right)$.

Theorem 1: Consider the system (7) in closed loop with the controller (12)-(13) with $K_{d t}, K_{p t}, K_{d \theta}$, and $K_{p \theta}$ diagonal positive definite and let Assumption 1 hold. Suppose, in addition, that there exists $T^{*}>0$ such that $T_{i j}(t) \in\left[0, T^{*}\right]$ for all $i, j \leq N, t \geq 0$ and the matrices $K_{d t}$ and $K_{p t}$ satisfy

$$
1-\left(1+N^{2} \bar{a}^{2}\right) T^{*} \lambda_{M}\left(K_{p t} K_{d t}^{-1}\right) \geq 0,
$$

where $\lambda_{M}(\cdot)$ denotes the largest eigenvalue, $\bar{a}:=\max \left\{a_{i j}\right\}$. Then, the origin in the closed-loop state space, i.e, $\{(e, s, \tilde{\theta}, v, \omega)=$ $(0,0,0,0,0)\}$, is uniformly globally asymptotically stable.

The proof of Theorem 1 is based on Lyapunov's direct method. For clarity of exposition, it is divided in two main parts: first, we derive the closed-loop equations and, then, we present the main guidelines to construct a strict Lyapunov-Krasovskiur functional.

\section{B. The closed-loop equations}

The first two error equations are obtained by differentiating on both sides of the the two equations in (10) and using (7a) and

$$
\dot{\Phi}(\theta)=-\Phi(\theta)^{\perp} \bar{\omega}, \quad \dot{\Phi}(\theta)^{\perp}=\Phi(\theta) \bar{\omega},
$$

where $\bar{\omega}:=\operatorname{diag}\left[\omega_{i}\right] \in \mathbb{R}^{N \times N}$, to obtain

$$
\begin{aligned}
\dot{e} & =-\bar{\omega} s+\Phi(\theta)^{\top} \mathcal{L} \Phi(\theta) v \\
\dot{s} & =\bar{\omega} e+\Phi(\theta)^{\perp \top} \mathcal{L} \Phi(\theta) v .
\end{aligned}
$$

Then, by direct substitution of (12) and (13) in (7b) and (7d), respectively, we obtain

$$
\begin{aligned}
\dot{v} & =-K_{d t} v-K_{p t} e_{d} \\
\dot{\tilde{\theta}} & =\omega \\
\dot{\omega} & =-K_{d \theta} \omega-K_{p \theta} \tilde{\theta}-p(t) \kappa\left(s_{d}, e_{d}\right) .
\end{aligned}
$$

Note the dependence on the delayed errors $e_{d}$ and $s_{d}$ in (23a) and (23c) - for the purpose of analysis we stress that, after (14) and (15), $e_{d}$ and $s_{d}$ may be expressed in function of $e$ and $s$ as

$$
\begin{aligned}
& e_{d}=e+\Phi(\theta)^{\top} \mathcal{A}\left(\dot{z}_{t}\right), \\
& s_{d}=s+\Phi(\theta)^{\perp \top} \mathcal{A}\left(\dot{z}_{t}\right),
\end{aligned}
$$

where $\dot{z}_{t}$ is short notation for $\dot{z}_{t}(\Delta):=\dot{z}(t+\Delta)$, with $\Delta \in\left[-T^{*}, 0\right]$ and the vector $\mathcal{A}\left(\dot{z}_{t}\right) \in \mathbb{R}^{2 N}$ is defined as

$$
\mathcal{A}\left(\dot{z}_{t}\right):=\left[\begin{array}{c}
\mathcal{A}_{1}\left(\dot{z}_{t}\right) \\
\vdots \\
\mathcal{A}_{N}\left(\dot{z}_{t}\right)
\end{array}\right], \quad \mathcal{A}_{i}\left(\dot{z}_{t}\right)=\sum_{j \in \mathcal{N}_{i}} a_{i j} \int_{t-T_{j i}(t)}^{t} \dot{z}_{j}(\tau) d \tau .
$$

Furthermore, $\kappa\left(e_{d}, s_{d}\right)$ in (23c) may be expressed as

$$
\kappa\left(e_{d}, s_{d}\right)=\kappa(e, s)+\kappa_{d}\left(e, s, \theta, \dot{z}_{t}\right)
$$

where

$$
\kappa_{d}=\frac{1}{2}\left[\begin{array}{c}
\mathcal{A}_{1}^{\top} \mathcal{A}_{1}+2 e_{1} \phi_{1}^{\top} \mathcal{A}_{1}+2 s_{1} \phi_{1}^{\perp \top} \mathcal{A}_{1} \\
\vdots \\
\mathcal{A}_{N}^{\top} \mathcal{A}_{N}+2 e_{N} \phi_{N}^{\top} \mathcal{A}_{N}+2 s_{N} \phi_{N}^{\perp \top} \mathcal{A}_{N}
\end{array}\right] .
$$

Remark 4: If measurements are not affected by delays, $\mathcal{A}_{i}^{\top}\left(\dot{z}_{t}\right)=0$ and we recover the error-dynamics equations from [14], [22].

Remark 5: The smooth time-varying stabilization mechanism may be roughly explained as follows. On one hand, the term $-p(t) \kappa(s, e)$ acts as a perturbation to the angular dynamics, $(23 \mathrm{c})$, as long as either of the errors, $e_{d}$ and $s_{d}$ are away from a $\delta$-neighbourhood of the origin. This is necessary because without the term $-p(t) \kappa\left(s_{d}, e_{d}\right)$, even in the absence of delays, $\theta$ would converge exponentially fast to the reference $\theta_{d}$. Now, on $\{v=0, \omega=0\}$, we have $\dot{e}(t)=\dot{s}(t)=0$ - see (21), (22) without guarantee that consensus has been reached, i.e., that both, $e(t)=0$ and $s(t)=0$-see Eq. (11). On the other hand, the control input $u_{v}$ steers $v, e_{d}$, and $s_{d}$ to zero, rendering the "perturbation" $-p(t) \kappa\left(s_{d}, e_{d}\right)$ smaller in norm and thereby allowing for $\tilde{\theta}$ to converge to zero as well. For further insight on $\delta$-persistentlyexciting controllers see [23], [28], [14].

Next, akin to latter reference, for the purpose of analysis let us introduce the new errors

$$
e_{\theta}=\tilde{\theta}+q(t) \kappa(s, e), \quad e_{\omega}=\omega+\dot{q}(t) \kappa(s, e)
$$

where $q: \mathbb{R}_{\geq 0} \rightarrow \mathbb{R}^{N \times N}$ is a differentiable function defined dynamically as

$$
\ddot{q}+K_{d \theta} \dot{q}+K_{p \theta} q=p(t) I_{N},
$$

where $K_{d \theta}$ and $K_{p \theta}$ are diagonal positive definite matrices and $I_{N}$ denotes the $N \times N$ identity matrix. Therefore, in view of (18) there exists $b_{q}>0$ such that

$$
\max \left\{|q|_{\infty},|\dot{q}|_{\infty},|\ddot{q}|_{\infty},\left|q^{(3)}\right|_{\infty}\right\} \leq b_{q}
$$

In the new equivalent coordinates (27), the closed-loop dynamics equations become

$$
\begin{array}{r}
\dot{X}_{t}=\left[\begin{array}{ccc}
-K_{d t} & -K_{p t} & 0 \\
0 & 0 & \dot{q} \bar{\kappa}-\bar{e}_{\omega} \\
0 & -\dot{q} \bar{\kappa}+\bar{e}_{\omega} & 0
\end{array}\right] X_{t} \\
+\left[\begin{array}{c}
0 \\
\Phi^{\top} \mathcal{L} \\
\Phi^{\perp \top} \mathcal{L}
\end{array}\right] \Phi v-\left[\begin{array}{c}
K_{p t} \Phi^{\top} \\
0 \\
0
\end{array}\right] \mathcal{A}\left(\dot{z}_{t}\right) \quad(30 \mathrm{a}) \\
\dot{X}_{r}=\left[\begin{array}{cc}
0 & I_{N} \\
-K_{p \theta} & -K_{d \theta}
\end{array}\right] X_{r}+\left[\begin{array}{l}
q \\
\dot{q}
\end{array}\right]\left[\bar{e} \Phi^{\top}+\bar{s} \Phi^{\perp \top}\right] \mathcal{L} \Phi v-\left[\begin{array}{l}
0 \\
p
\end{array}\right] \kappa_{d},
\end{array}
$$

where $\bar{\kappa}:=\operatorname{diag}\left[s_{i}^{2}+e_{i}^{2}\right], \bar{e}_{\omega}:=\operatorname{diag}\left[e_{\omega_{i}}\right], X_{t}:=\left[v^{\top} e^{\top} s^{\top}\right]^{\top}$, and $X_{r}:=\left[\begin{array}{ll}e_{\theta}^{\top} & e_{\omega}^{\top}\end{array}\right]^{\top}$. The rest of the proof consists in establishing uniform global asymptotic stability of the origin for (30).

\section{Stability analysis}

We proceed now to construct a Lyapunov-Krasovskiĭ functional for (30), by relying on the Lyapunov function proposed in [22] for the system (21)-(23) subject to $\left(e_{d}, s_{d}\right)=(e, s)$, that is, without delays.

First, we observe that the translational part of the system, i.e., Eq. (30a), admits the following non-strict Lyapunov-Krasovskii functional

$$
V\left(v, z, \dot{z}_{t}\right)=v^{\top} K_{p t}^{-1} v+z^{\top} \mathcal{L} z+\int_{-T^{*}}^{0} \int_{t+\theta}^{t}|\dot{z}(\tau)|^{2} d \tau d \theta .
$$


After [14, Lemma 1], we have

$$
\lambda_{2}(L) z^{\top} \mathcal{L} z \leq|e|^{2}+|s|^{2} \leq \lambda_{N}(L) z^{\top} \mathcal{L} z,
$$

where $\lambda_{i}(L)$ denotes the $i$ th eigenvalue of $L$ so, in view of the inequality

$$
\int_{-T^{*}}^{0} \int_{t+\theta}^{t}|\dot{z}(\tau)|^{2} d \tau d \theta \leq T^{*} \int_{t-T^{*}}^{t}|\dot{z}(\tau)|^{2} d \tau,
$$

it follows, according to [29], that the functional $V$ is positive definite and radially unbounded with respect to $X_{t}$. Furthermore, the timederivative of $V$ along the trajectories of (30a) is

$$
\begin{aligned}
\dot{V}= & -2 v^{\top} K_{p t}^{-1} K_{d t} v+2 v^{\top} \Phi(\theta)^{\top} \mathcal{A}\left(\dot{z}_{t}\right)+T^{*}|v|^{2} \\
& -\int_{t-T^{*}}^{t}|\dot{z}(\tau)|^{2} d \tau \\
\leq & -\left[2-T^{*} \lambda_{M}\left(K_{p t} K_{d t}^{-1}\right)\right] v^{\top} K_{p t}^{-1} K_{d t} v+2 v^{\top} \Phi(\theta)^{\top} \mathcal{A}\left(\dot{z}_{t}\right) \\
& -\frac{1}{\bar{a}^{2} N} \sum_{j=1}^{N} \sum_{i=1}^{N} a_{i j}^{2} \int_{t-T_{i j}}^{t}\left|\dot{z}_{i}(\tau)\right|^{2} d \tau .
\end{aligned}
$$

Now, on one hand, after Jensen's inequality, we have

$$
-\int_{t-T_{i j}}^{t}\left|\dot{z}_{i}(\tau)\right|^{2} d \tau \leq-\frac{1}{T_{i j}^{*}}\left|\int_{t-T_{i j}}^{t} \dot{z}_{i}(\tau) d \tau\right|^{2}
$$

while, on the other, the second term on the right-hand side of (33) satisfies $2 v^{\top} \Phi(\theta)^{\top} \mathcal{A}\left(\dot{z}_{t}\right) \leq(N / 2 \epsilon)\left|\mathcal{A}\left(\dot{z}_{t}\right)\right|^{2}+$ $\epsilon \lambda_{M}\left(K_{p t} K_{d t}^{-1}\right) v^{\top} K_{p t}^{-1} K_{d t} v$ for any $\epsilon>0$. Let $\epsilon=N^{2} \bar{a} T^{*}$. Then, using

$$
\left|\mathcal{A}\left(\dot{z}_{t}\right)\right|^{2} \leq N \sum_{j=1}^{N} \sum_{i=1}^{N} a_{i j}^{2}\left|\int_{t-T_{i j}}^{t} \dot{z}_{i}(\tau) d \tau\right|^{2}
$$

and (19), we obtain

$$
\begin{aligned}
\dot{V} & \leq-v^{\top} K_{p t}^{-1} K_{d t} v-Y\left(\dot{z}_{t}\right) \\
Y\left(\dot{z}_{t}\right) & :=\frac{1}{2 \bar{a}^{2} N T^{*}} \sum_{j=1}^{N} \sum_{i=1}^{N} a_{i j}^{2}\left|\int_{t-T_{i j}}^{t} \dot{z}_{i}(\tau) d \tau\right|^{2}
\end{aligned}
$$

which is negative semidefinite. That is, $V$ is a non-strict LyapunovKrasovskil functional for the system (30a).

Next, following the Mazenc construction method of strict Lyapunov functions [25], [24], we introduce a function $\left(t, X_{t}, V\right) \mapsto$ $W\left(t, X_{t}, V\right)$. We recall the function proposed in [22]:

$$
\begin{aligned}
W= & \gamma(V) V+V \kappa(s, e)^{\top} \Upsilon(t) \kappa(s, e)+\alpha(V) e^{\top} v-c_{1} V e^{\top} \dot{q} s \\
& +c_{1} b_{q} \lambda_{N}(L) V^{2}+\left[\lambda_{N}(L)+\left|K_{p t}\right|\right] \alpha(V) V
\end{aligned}
$$

where $b_{q}>0$ is defined in (29), $\gamma$ and $\alpha$ are non decreasing functions mapping $\mathbb{R}_{\geq 0}$ to $\mathbb{R}_{>0}$ chosen as polynomials of $V$ - see the appendix for details-, and

$$
\Upsilon(t):=1+2 b_{q}^{2} T I_{N}-\frac{2}{T} \int_{t}^{t+T} \int_{t}^{m} \dot{q}(\tau)^{2} d \tau d m .
$$

Remark 6: We stress for further development that $e^{\top} v$ and $e^{\top} s$ are quadratic terms that, in view of the triangle inequality, (31), and (32), satisfy $e^{\top} v+e^{\top} s \leq c V$ for some appropriate value of $c>0$. Therefore, in view of (29),

$$
\alpha(V) e^{\top} v-c_{1} V e^{\top} \dot{q} s \leq c \alpha^{\prime}(V)
$$

for some continuous non-decreasing function $\alpha^{\prime}: \mathbb{R}_{\geq 0} \rightarrow \mathbb{R}_{>0}$. The specific definitions of $c$ and $\alpha^{\prime}$ are irrelevant, but in the sequel we use extensively this type of bound.

The computation of $\dot{W}$ involves lengthy but straightforward steps that are relegated to the appendix $-c f$. [21]. We rather underline the purpose of the terms that compose $W$ and the main steps in the differentiation of this function.

The purpose of the term $\gamma(V) V$ is to generate in $\dot{W}$ the term $-\gamma(V) v^{\top} K_{p t}^{-1} K_{d t} v$, whose negativity is "enforced" by the factor $\gamma(V)-c f$. (36). The second term on the right-hand side of (37), generates the negative term $-\frac{\mu}{T} V|\kappa(e, s)|^{2}$. This is in virtue of the fact that

$$
\dot{\Upsilon}(t) \leq-\frac{2}{T} \int_{t}^{t+T} \dot{q}(\tau)^{2} d \tau+2 b_{q}^{2} I_{N} \quad \forall t \geq 0
$$

and $\dot{q}$ is persistently exciting, as per Assumption 1. Indeed, this follows because $\dot{q}$ is solution to

$$
q^{(3)}+K_{d \theta} \ddot{q}+K_{p \theta} \dot{q}=\dot{p}(t) I_{N}
$$

-cf. (28), [30].

Next, the respective derivatives of the third and fourth terms on the right hand side of (37) satisfy, respectively,

$$
\begin{aligned}
\frac{d}{d t}\left[\alpha(V) e^{\top} v\right] \leq & -\frac{\partial \alpha}{\partial V} e^{\top} v v^{\top} K_{d t} K_{p t}^{-1} v+\alpha(V)\left[v^{\top} \Phi^{\top} \mathcal{L} \Phi v\right. \\
& \left.+s^{\top} \dot{q} \bar{\kappa} v-e^{\top} K_{d t} v-e^{\top} K_{p t} e-v^{\top} \bar{e}_{\omega} s\right] \\
\frac{d}{d t}\left[-c_{1} V e^{\top} \dot{q} s\right] \leq & -e^{\top} \ddot{q} s V c_{1}+c_{1}\left[e^{\top} \dot{q} s\right] v^{\top} K_{d t} K_{p t}^{-1} v \\
& -c_{1} V\left[v^{\top} \Phi^{\top} \mathcal{L} \Phi \dot{q} s+s^{\top} \bar{\kappa} \dot{q}^{2} s-e^{\top} \dot{q}^{2} \bar{\kappa} e\right. \\
& \left.+e^{\top} \dot{q} \Phi^{\perp \top} \mathcal{L} \Phi v-s^{\top} \bar{e}_{\omega} \dot{q} s+e^{\top} \dot{q} \bar{e}_{\omega} e\right] .
\end{aligned}
$$

Note the non-positive terms $-\alpha(V) e^{\top} K_{p t} e$ and $-c_{1} V s^{\top} \bar{\kappa} \dot{q}^{2} s$ in (41) and (42) respectively; all the other terms are of undefined sign and may be bounded as those in (39). On the other hand, in view of (36), the total derivative of the last two terms that define $W$ is negative semidefinite and serves in bounding from above the terms of undefined sign in (41) and (42). Thus, for an appropriate choice of $\gamma(V)$ and $\alpha(V)$ - see the appendix for details-, we obtain

$$
\begin{aligned}
\dot{W} \leq & -\frac{1}{2} \gamma(V) v^{\top} K_{d t} K_{p t}^{-1} v-\frac{1}{4} \alpha(V) e^{\top} K_{p t} e-\frac{\mu}{2 T} V|\kappa(s, e)|^{2} \\
& -\alpha(V) v^{\top} \bar{e}_{\omega} s+c_{1} V s^{\top} \dot{q} \bar{e}_{\omega} s-\dot{q} c_{1} V e^{\top} \dot{q} \bar{e}_{\omega} e \\
& -\alpha(V) e^{\top} K_{p t} \Phi(\theta)^{\top} \mathcal{A}\left(\dot{z}_{t}\right) \\
& -\left[\gamma(V)-c_{1} e^{\top} \dot{q} s+\left[\frac{\partial \alpha}{\partial V} e^{\top} v\right]\right] Y\left(\dot{z}_{t}\right) .
\end{aligned}
$$

Now, for any $\sigma>0$ we have

$$
\begin{gathered}
\alpha(V) v^{\top} \bar{e}_{\omega} s \leq \frac{\lambda_{N}(L)\left|K_{d t}^{-1} K_{p t}\right|}{\sigma} \alpha(V) V v^{\top} K_{d t} K_{p t}^{-1} v+\frac{\sigma}{4} \alpha(V)\left|e_{\omega}\right|^{2}, \\
c_{1} V e_{\omega}^{\top} \dot{q}(\bar{s} s-\bar{e} e) \leq c_{1} V b_{q}\left[\frac{\sigma}{4}\left|e_{\omega}\right|^{2}+\frac{4}{\sigma}|\kappa(s, e)|^{2}\right]
\end{gathered}
$$

Furthermore,

$-\alpha(V) e^{\top} K_{p t} \Phi(\theta)^{\top} \mathcal{A}\left(\dot{z}_{t}\right) \leq \frac{\alpha(V)}{16} e^{\top} K_{p t} e+4 \alpha(V) \lambda_{M}\left(K_{p t}\right)|\mathcal{A}|^{2}$,

and

$$
|\mathcal{A}|^{2} \leq N \sum_{j=1}^{N} \sum_{i=1}^{N} a_{i j}^{2}\left|\int_{t-T_{i j}}^{t} \dot{z}_{i}(\tau) d \tau\right|^{2}
$$

hence, $\left|\mathcal{A}\left(\dot{z}_{t}\right)\right|^{2} \leq 2 N^{2} T^{*} \bar{a}^{2} Y\left(\dot{z}_{t}\right)$ and for an appropriate choice of $\gamma(V)$ and $\alpha(V)$, the sum of the last two terms on the right-hand side of (43) is non-positive. Furthermore, after (44)-(45), if we set

$$
\sigma(V)=\frac{4 \lambda_{N}(L)\left|K_{d t}^{-1} K_{p t}\right| \alpha(V) V}{\gamma(V)}+\frac{16 T c_{1} b_{q}}{\mu}
$$

we obtain

$$
\dot{W} \leq-\frac{1}{4} \gamma(V) v^{\top} K_{d t} K_{p t}^{-1} v-\frac{1}{8} \alpha(V) e^{\top} K_{p t} e
$$




$$
-\frac{\mu}{4 T} V|\kappa(s, e)|^{2}+\frac{\sigma}{4}\left[\alpha(V)+b_{q} c_{1} V\right]\left|e_{\omega}\right|^{2} .
$$

Note that, apart from the last term on the right-hand side of (47), $\dot{W}$ is negative definite in $X_{t}$.

The next step in the construction of a Lyapunov-Krasovskil functional for the closed-loop system is to design a function of $X_{r}$ whose derivative contains terms that are sufficiently negative to dominate over the last term on the right-hand side of (47). Let

$$
Z\left(X_{r}\right)=c_{2}\left[e_{\omega}^{\top} e_{\omega}+e_{\theta}^{\top} K_{p \theta} e_{\theta}\right]+e_{\theta}^{\top} e_{\omega}
$$

which is positive definite in $X_{r}$ for an appropriate choice of $c_{2}>0$. For clarity of exposition in the computation of $\dot{Z}$ along the trajectories of (30b) let, for the time being, $\kappa_{d} \equiv 0$. Then, the derivative of $Z$ along the trajectories of (30b) satisfies

$$
\begin{aligned}
\dot{Z}\left(X_{r}\right) \leq & -\left[\frac{c_{2}}{4} e_{\omega}^{\top} K_{d \theta} e_{\omega}+\frac{1}{4} e_{\theta}^{\top} K_{p \theta} e_{\theta}\right] \\
& +\frac{c_{2}}{2}\left|\bar{e} \Phi^{\top} \mathcal{L} \Phi v\right|^{2}+\frac{c_{2}}{2}\left|\bar{s} \Phi^{\perp \top} \mathcal{L} \Phi v\right|^{2}
\end{aligned}
$$

Furthermore, using the inequalities

$$
\begin{gathered}
\left|\bar{e} \Phi^{\top} \mathcal{L} \Phi v\right|^{2} \leq \lambda_{N}(L) V\left|\Phi^{\top} \mathcal{L} \Phi\right|_{\infty}^{2}\left|K_{d t}^{-1} K_{p t}\right| v^{\top} K_{d t} K_{p t}^{-1} v \\
\left|\bar{s} \Phi^{\perp \top} \mathcal{L} \Phi v\right|^{2} \leq \lambda_{N}(L) V\left|\Phi^{\perp \top} \mathcal{L} \Phi\right|_{\infty}^{2}\left|K_{d t}^{-1} K_{p t}\right| v^{\top} K_{d t} K_{p t}^{-1} v
\end{gathered}
$$

and defining

$$
\rho_{3}(V)=\frac{c_{2} \lambda_{N}(L)\left|K_{d t}^{-1} K_{p t}\right|}{2}\left[\left|\Phi^{\top} \mathcal{L} \Phi\right|_{\infty}^{2}+\left|\Phi^{\perp \top} \mathcal{L} \Phi\right|_{\infty}^{2}\right],
$$

we obtain

$\dot{Z}\left(X_{r}\right) \leq-\left[\frac{c_{2}}{4} e_{\omega}^{\top} K_{d \theta} e_{\omega}+\frac{1}{4} e_{\theta}^{\top} K_{p \theta} e_{\theta}\right]+2 \rho_{3}(V) V v^{\top} K_{d t} K_{p t}^{-1} v$.

By a simple inspection one may see that $\dot{W}+[\cdot] \dot{Z}$ can be rendered negative semidefinite if $Z\left(X_{r}\right)$ is scaled appropriately by a factor depending on $V$. Let $V \mapsto \rho_{1}(V)$ be a strictly increasing function of $V$ such that $\rho_{1}(0)>0$. Then, the total derivative of $\rho_{1}(V) Z\left(X_{r}\right)$ along the trajectories of $(30 \mathrm{~b})$ yields

$$
\begin{aligned}
\frac{d}{d t}\left[\rho_{1}(V) Z\left(X_{r}\right)\right] \leq & -\rho_{1}(V)\left[\frac{c_{2}}{4} e_{\omega}^{\top} K_{d \theta} e_{\omega}+\frac{1}{4} e_{\theta}^{\top} K_{p \theta} e_{\theta}\right] \\
+ & \rho_{1}(V) \rho_{3}(V) V v^{\top} K_{d t} K_{p t}^{-1} v+2 \rho_{1}(V) K_{p t} c_{2} e_{\omega}^{\top} \kappa_{d} \\
& +\rho_{1}(V) K_{p t} e_{\theta}^{\top} \kappa_{d}+Z\left(X_{r}\right) \dot{V}
\end{aligned}
$$

and we recall that $\dot{V}$ satisfies (36). Then, we use the identity

$$
\kappa_{d}\left(e_{d}, s_{d}\right)=\frac{1}{2}\left[\begin{array}{c}
\left|\mathcal{A}_{1}\right|^{2} \\
\vdots \\
\left|\mathcal{A}_{N}\right|^{2}
\end{array}\right]+\bar{e} \Phi^{\top} \mathcal{A}\left(\dot{z}_{t}\right)+\bar{s} \Phi^{\perp \top} \mathcal{A}\left(\dot{z}_{t}\right)
$$

as well as the inequalities

$$
\begin{aligned}
& \lambda_{M}\left(K_{p t}\right) c_{2} \rho_{1}(V)\left|e_{\omega}\right||\mathcal{A}|^{2} \leq \frac{\lambda_{M}\left(K_{p t}\right) c_{2}}{2 \epsilon_{1}}\left|e_{\omega}\right|^{2}|\mathcal{A}|^{2} \\
&+\lambda_{M}\left(K_{p t}\right) c_{2} \rho_{1}(V)^{2} \frac{\epsilon_{1}}{2}|\mathcal{A}|^{2}, \\
& \frac{1}{2} \lambda_{M}\left(K_{p t}\right) \rho_{1}(V)\left|e_{\theta}\right||\mathcal{A}|^{2} \leq \frac{\lambda_{M}\left(K_{p t}\right)}{4 \epsilon_{1}}\left|e_{\theta}\right|^{2}|\mathcal{A}|^{2} \\
&+\lambda_{M}\left(K_{p t}\right) \rho_{1}(V)^{2} \frac{\epsilon_{1}}{4}|\mathcal{A}|^{2}, \\
& 2 \rho_{1}(V) K_{p t} c_{2} e_{\omega}^{\top}\left[\bar{e} \Phi^{\top}+\bar{s} \Phi^{\perp \top}\right] \mathcal{A}\left(\dot{z}_{t}\right) \leq 2 \frac{\lambda_{M}\left(K_{p t}\right) \rho_{1}(V) c_{2}}{\epsilon}\left|e_{\omega}\right|^{2} \\
&+\epsilon \lambda_{M}\left(K_{p t}\right) c_{2} \rho_{1}(V)\left(e^{2}+s^{2}\right)|\mathcal{A}|^{2}, \\
& \rho_{1}(V) K_{p t} e_{\theta}^{\top}\left[\bar{e} \Phi^{\top}+\bar{s} \Phi^{\perp \top}\right] \mathcal{A}\left(\dot{z}_{t}\right) \leq \frac{\lambda_{M}\left(K_{p t}\right) \rho_{1}(V)}{\epsilon}\left|e_{\theta}\right|^{2}
\end{aligned}
$$

$$
+\rho_{1}(V) \lambda_{M}\left(K_{p t}\right)\left(e^{2}+s^{2}\right) \frac{\epsilon}{2}|\mathcal{A}|^{2},
$$

which hold for any $\epsilon, \epsilon_{1}>0$, to obtain

$$
\begin{aligned}
\frac{d}{d t}\left[\rho_{1}(V) Z\left(X_{r}\right)\right] \leq-\rho_{1}(V)\left[\frac{c_{2}}{4} e_{\omega}^{\top} K_{d \theta} e_{\omega}+\frac{1}{4} e_{\theta}^{\top} K_{p \theta} e_{\theta}\right] \\
+\rho_{1}(V) \rho_{3}(V) V v^{\top} K_{d t} K_{p t}^{-1} v+Z\left(X_{r}\right) \dot{V} \\
\quad+\frac{\lambda_{M}\left(K_{p t}\right) \rho_{1}(V)}{\epsilon}\left[2 c_{2}+1\right]\left[\left|e_{\omega}\right|^{2}+\left|e_{\theta}\right|^{2}\right] \\
\quad+\frac{\lambda_{M}\left(K_{p t}\right)}{2 \epsilon_{1}}\left[c_{2}+\frac{1}{2}\right]\left[\left|e_{\omega}\right|^{2}+\left|e_{\theta}\right|^{2}\right]|\mathcal{A}|^{2} \\
+\lambda_{M}\left(K_{p t}\right) \rho_{1}(V)\left[c_{2}+\frac{1}{2}\right]\left[\rho_{1}(V) \frac{\epsilon_{1}}{2}+\lambda_{N}(L) V \epsilon\right]|\mathcal{A}|^{2} .
\end{aligned}
$$

We see that for sufficiently large values of $\epsilon$, the sum of the first and fourth terms on the right-hand side of (51) is non-positive and so is the sum of the third and fifth terms, in view of (36) and

$$
\begin{gathered}
Z\left(X_{r}\right) \geq \min \left\{1, \lambda_{m}\left(K_{p \theta}\right)\right\}\left[\left|e_{\theta}\right|^{2}+\left|e_{\omega}\right|^{2}\right], \\
|\mathcal{A}|^{2} \leq N \sum_{j=1}^{N} \sum_{i=1}^{N} a_{i j}^{2}\left|\int_{t-T_{i j}}^{t} \dot{z}_{i}(\tau) d \tau\right|^{2} .
\end{gathered}
$$

Therefore, we have

$$
\begin{aligned}
\frac{d}{d t}\left[\rho_{1}(V) Z\left(X_{r}\right)\right] \leq-\rho_{1}(V)\left[\frac{3 c_{2}}{16} e_{\omega}^{\top} K_{d \theta} e_{\omega}+\frac{3}{16} e_{\theta}^{\top} K_{p \theta} e_{\theta}\right] \\
+\lambda_{M}\left(K_{p t}\right) \rho_{1}(V)\left[c_{2}+\frac{1}{2}\right]\left[\rho_{1}(V) \frac{\epsilon_{1}}{2}+\lambda_{N}(L) V \epsilon\right]|\mathcal{A}|^{2} \\
+\rho_{1}(V) \rho_{3}(V) V v^{\top} K_{d t} K_{p t}^{-1} v .
\end{aligned}
$$

Thus, in view of (47) and (52), the total derivative of

$$
\mathcal{V}\left(t, X_{t}, X_{r}\right)=W(t, V, v, e, s)+\rho_{1}(V) Z\left(X_{r}\right)+\rho_{2}(V) V
$$

along the trajectories of (30), where

$$
\begin{aligned}
\rho_{2}(V)= & 2 \lambda_{M}\left(K_{p t}\right) \rho_{1}(V)\left[1+c_{2}\right] N^{2} \bar{a}^{2} T^{*}\left[\lambda_{N}(L) \epsilon V+\epsilon_{1} \rho_{1}(V)\right] \\
& +2 \rho_{1}(V) \rho_{3}(V) V
\end{aligned}
$$

satisfies

$$
\begin{aligned}
\dot{\mathcal{V}} \leq- & \frac{1}{8} \gamma(V) v^{\top} K_{d t} K_{p t}^{-1} v-\frac{1}{16} \alpha(V) e^{\top} K_{p t} e-\frac{\mu}{8 T} V|\kappa(s, e)|^{2} \\
& -\rho_{1}(V)\left[\frac{c_{2}}{16} e_{\omega}^{\top} K_{d \theta} e_{\omega}+\frac{1}{16} e_{\theta}^{\top} K_{p \theta} e_{\theta}\right]
\end{aligned}
$$

which is negative definite since, by design, $\rho_{1}(0)>0$. Also, $\mathcal{V}\left(t, X_{t}, X_{r}\right)$ is positive definite. The result follows.

\section{Simulation RESUlts}

We present now some illustrative numerical simulations using four differential wheeled mobile robots. The desired formation pattern is fixed to be a rhomboid with an open-chain interconnection graph; its Laplacian and schematic representation are given in Figure 1.

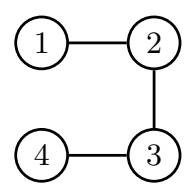

$$
L=\left[\begin{array}{rrrr}
1 & -1 & 0 & 0 \\
-1 & 2 & -1 & 0 \\
0 & -1 & 2 & -1 \\
0 & 0 & -1 & 1
\end{array}\right]
$$

Fig. 1. Interconnection undirected graph and Laplacian matrix

The initial positions and the relative distances with regards to the center of the formation are given in Table I. The control gains have been set to: $K_{d t}=60 \mathbf{I}_{4} ; K_{p t}=30 \mathbf{I}_{4} ; K_{d \theta}=30 \mathbf{I}_{4}$; and $K_{p \theta}=10 \mathbf{I}_{4}$. The persistently exciting function $p(t)$ is given by 
$p(t)=-3+2 \pi \sin (0.5 t)$. For simplicity, the time-varying delay is the same for all the interconnections and is depicted in Fig. 2, its bound has been taken as $T^{*}=0.37$. These delays follow a normal Gaussian distribution with a mean of 0.3 and a variance of 0.0003 — $c f$. [31]. Under this scenario, condition (19) is satisfied.

TABLE I

INITIAL CONDITIONS AND DESIRED ORIENTATIONS AND RELATIVE

\begin{tabular}{c|c|c|c|c|c|c|}
\cline { 2 - 7 } & $x_{i}(0)$ & $y_{i}(0)$ & $\theta_{i}(0)$ & $\delta_{x_{i}}$ & $\delta_{y_{i}}$ & $\theta_{d i}$ \\
\hline 1 & 5 & -7 & $-(1 / 3) \pi$ & -2 & 0 & -1 \\
\hline 2 & -4 & 7 & $(2 / 3) \pi$ & 0 & 2 & 1 \\
\hline 3 & -2 & -5 & $(4 / 3) \pi$ & 2 & 0 & 0 \\
\hline 4 & 7 & 3 & 0 & 0 & -2 & 0.5 \\
\hline
\end{tabular}

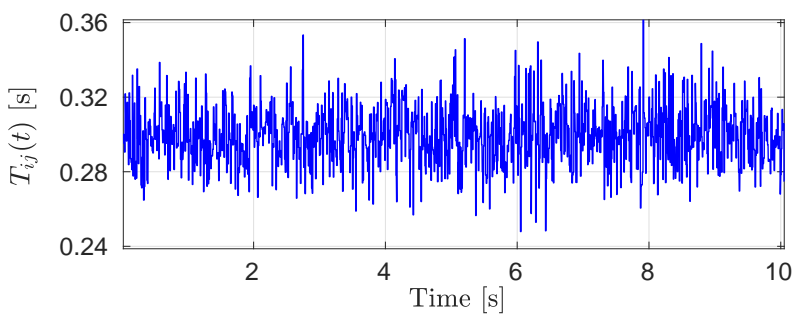

Fig. 2. Time delay in the network interconnection.

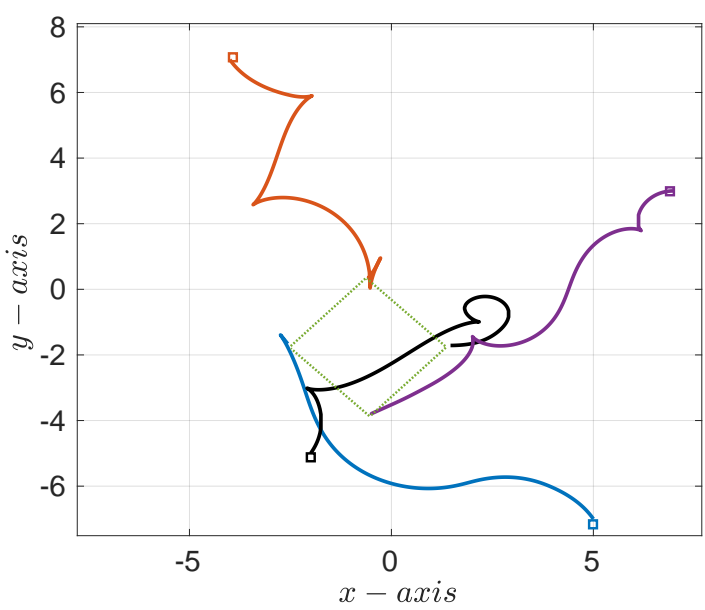

Fig. 3. Trajectories and formation of the network of mobile robots.

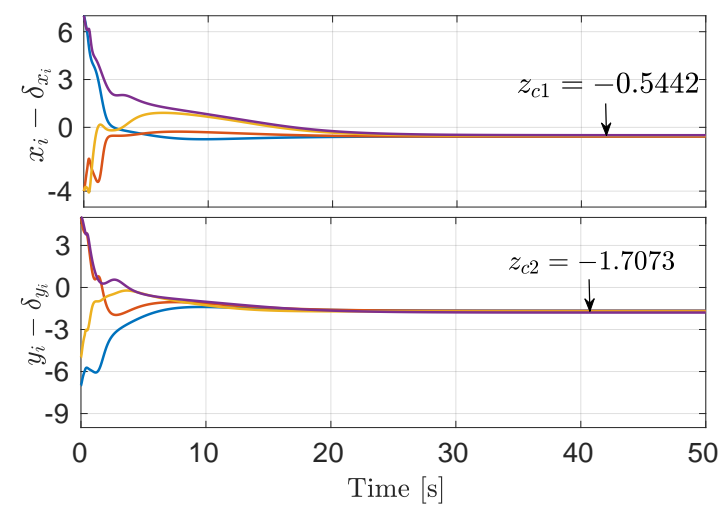

Fig. 4. Evolution of the relative positions $z_{i}$.

Fig. 3 shows the $x_{i}, y_{i}$ trajectories of the nonholonomic mobile robots, while Figs. 4 and 5 depict the relative positions $z_{i}$ and the orientation of the robots. From these plots it can be observed that the robots reach the target formation and their respective orientations converge to the desired values. The "zigzaggy" motion is common in stabilization of nonholonomic robots — $c$. [9], [6], [11], [15].

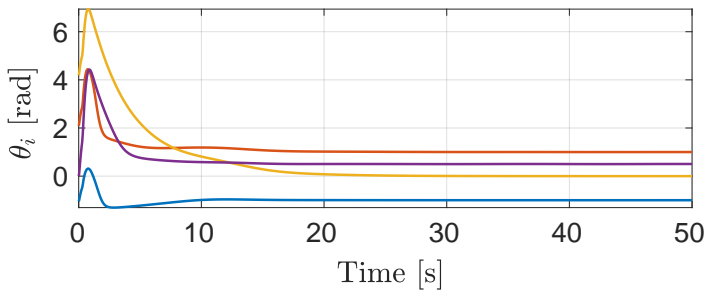

Fig. 5. Orientation of each mobile robot in the network.

\section{Conclusions}

A decentralized smooth time-varying controller that guarantees partial-consensus among groups of nonholonomic mobile robots interacting over a network that is affected by time-varying communication delays was presented. Uniform global asymptotic stability of the consensus set is formally established via constructive proof (a strict Lyapunov-Krasovskiı functional is provided). From a practical viewpoint, however, the solution presented has the limitation of relying on global-positioning systems which, in a variety of concrete robotics scenarii, are unavailable. Current research focuses on consensus control using relative-positioning measurements, including under the influence of delays.

\section{REFERENCES}

[1] W. Ren and R. W. Beard, Distributed consensus in multivehicle cooperative control. Springer verlag, 2005.

[2] Z. Li, W. Ren, X. Liu, and M. Fu, "Consensus of multi-agent systems with general linear and lipschitz nonlinear dynamics using distributed adaptive protocols," IEEE Trans. on Automatic Control, vol. 58, no. 7 , pp. 1786-1791, 2013.

[3] E. Panteley and A. Loría, "Synchronization and dynamic consensus of heterogeneous networked systems," IEEE Trans. on Automatic Control, vol. 62, no. 8, pp. 3758-3773, 2017.

[4] X. Zhao, X. Zheng, C. Ma, and R. Li, "Distributed consensus of multiple Euler-Lagrange systems networked by sampled-data information with transmission delays and data packet dropouts," IEEE Transactions on Automation Science and Engineering, vol. 14, no. 3, pp. 1440-1450, 2017.

[5] E. Nuño, I. Sarras, and L. Basañez, "Consensus in networks of nonidentical Euler-Lagrange systems using P+d controllers," IEEE Trans. on Robotics, vol. 26, no. 6, pp. 1503-1508, 2013.

[6] Z. Lin, B. Francis, and M. Maggiore, "Necessary and sufficient graphical conditions for formation control of unicycles," IEEE Trans. on Automatic Control, vol. 50, no. 1, pp. 121-127, 2005.

[7] R. W. Brockett, "Asymptotic stability and feedback stabilization," Differential geometric control theory, vol. 27, no. 1, pp. 181-191, 1983.

[8] D. A. Lizárraga., "Obstructions to the existence of universal stabilizers for smooth control systems," Mathematics of Control, Signals and Systems, vol. 16, p. 255277, 2004.

[9] A. Dong and J. A. Farrell, "Cooperative control of multiple nonholonomic mobile agents," IEEE Trans. on Automatic Control, vol. 53, no. 6, pp. 1434-1447, 2008.

[10] Z. Peng, G. Wen, A. Rahmani, and Y. Yu, "Distributed consensusbased formation control for multiple nonholonomic mobile robots with a specified reference trajectory," International Journal of Systems Science, vol. 46, no. 8, pp. 1447-1457, 2015.

[11] A. González, R. Aragüés, G. López-Nicolás, and C. Saguiés, "Stability analysis of nonholonomic multiagent coordinate-free formation control subject to communication delays," International Journal of Robust and Nonlinear Control, vol. 28, no. 14, pp. 4121-4138, 2018.

[12] N. Sarkar, X. Yun, and V. Kumar, "Control of mechanical systems with rolling constraints: application to dynamic control of mobile robots," International Journal of Robotics Research, vol. 13, no. 1, pp. 55-69, 1994. 
[13] K. D. Do, Z.-P. Jiang, and J. Pan, "A global output-feedback controller for simultaneous tracking and stabilization of unicycle-type mobile robots," IEEE Trans. on Robotics Automat., vol. 20, no. 3, pp. 589594, 2004.

[14] M. Maghenem, A. Bautista-Castillo, E. Nuño, A. Loría, and E. Panteley, "Consensus of multi-agent systems with nonholonomic restrictions via Lyapunov's direct method," IEEE Control Systems Letters, vol. 3, no. 2, pp. 344-349, 2019. DOI: 10.1109/LCSYS.2018.2879043.

[15] D. V. Dimarogonas and K. J. Kyriakopoulos, "On the rendezvous problem for multiple nonholonomic agents," IEEE Trans. on Automatic Control, vol. 52, no. 5, pp. 916-922, 2007.

[16] E. Nuño, A. Loría, A. T. Hernández, M. Maghenem, and E. Panteley, "Consensus-based formation control of nonholonomic robots with communication delays," Automatica, 2020. To appear (accepted in May 2020).

[17] M. Maghenem, A. Loriía, E. Nuño, and E. Panteley, "Distributed fullconsensus control of nonholonomic vehicles under non-differentiable measurement delays," IEEE Control Systems Letters, 2021. In Press. Pre-published online: https://ieeexplore.iee.org/document/9110600, DOI: $10.1109 /$ LCSYS.2020.3000676.

[18] K.-K. Oh, M.-C. Park, and H.-S. Ahn, "A survey of multi-agent formation control," Automatica, vol. 53, pp. 424-440, 2015.

[19] E. Nuño, T. Hernández, M. Maghenem, A. Loría, and E. Panteley, "Leaderless consensus-based formation control of multiple nonholonomic mobile robots with interconnecting delays," in Proc. IEEE American Control Conference, (Philadelphia, PA, USA), pp. 4659-4664, 2019. DOI: $10.23919 / \mathrm{ACC} .2019 .8814391$.

[20] A. Abdessameud, I. G. Polushin, and A. Tayebi, "Synchronization of Lagrangian systems with irregular communication delays," IEEE Trans. on Automatic Control, vol. 59, no. 1, pp. 187-193, 2014.

[21] M. Maghenem, Stability and stabilization of networked time-varying systems. PhD thesis, Univ Paris Saclay, Gif sur Yvette, 2017.

[22] M. Maghenem, A. Bautista-Castillo, E. Nuño, A. Loría and E. Panteley, "Consensus-based formation control of nonholonomic robots using a strict Lyapunov function," IFAC-PapersOnLine, vol. 50, no. 1, pp. 24392444, 2017. Presented at IFAC World Congress 2017, Toulouse, France. DOI: $10.1016 /$ j.ifacol.2017.08.406.

[23] A. Loría, E. Panteley, and K. Melhem, "UGAS of skew-symmetric timevarying systems: application to stabilization of chained form systems," European Journal of Control, vol. 8, no. 1, pp. 33-43, 2002.

[24] M. Malisoff and F. Mazenc, Constructions of Strict Lyapunov functions. London: Springer Verlag, 2009.

[25] F. Mazenc, "Strict Lyapunov functions for time-varying systems," Automatica, vol. 39, no. 2, pp. 349-353, 2003.

[26] N. N. Krasovskii, Problems of the theory of stability of motion. Stanford Univ. Press, 1963. Translation of Russian edition, Moscow 1959.

[27] A. Loría, E. Panteley, D. Popović, and A. Teel, "A nested Matrosov theorem and persistency of excitation for uniform convergence in stable non-autonomous systems," IEEE Trans. on Automatic Control, vol. 50, no. 2, pp. 183-198, 2005.

[28] A. Loría, E. Panteley, and A. Teel, "A new persistency-of-excitation condition for UGAS of NLTV systems: Application to stabilization of nonholonomic systems," in Proc. 5th. European Contr. Conf., (Karlsrühe, Germany), pp. 1363-1368, 1999.

[29] E. Fridman, "Tutorial on Lyapunov-based methods for time-delay systems," European Journal of Control, vol. 20, no. 6, pp. 271-283, 2014.

[30] P. Ioannou and J. Sun, Robust adaptive control. New Jersey, USA: Prentice Hall, 1996.

[31] P. Salvo-Rossi, G. Romano, F. Palmieri, and G. Iannello, "Joint endto-end loss-delay hidden markov model for periodic UDP traffic over the internet," IEEE Transactions on Signal Processing, vol. 54, no. 2, pp. 530-541, 2006.

\section{APPENDIX}

Detailed computation of $\dot{W}$, after (37)

We evaluate the total derivative of each term on the right-hand side of (37). First, since $\gamma$ is non-decreasing, we have

$$
\frac{d}{d t}[\gamma(V) V] \leq-\gamma(V) \dot{V}
$$

Now, for the second term, we have

$\frac{d}{d t}\left[V \kappa^{\top} \Upsilon(t) \kappa\right] \leq-V \kappa^{\top} \frac{1}{T} \int_{t}^{t+T} \dot{q}(s)^{2} d s \kappa+$

$$
V \kappa^{\top} \dot{q}(t)^{2} \kappa+2 V \kappa^{\top} \Upsilon\left[\bar{e} \Phi^{\top} \mathcal{L} \Phi v+\bar{s} \Phi^{\perp \top} \mathcal{L} \Phi v\right]
$$

and since $\dot{q}$ is persistently exciting with parameters $(\mu, T)$ and the following identities hold: $\kappa^{\top} \Upsilon \bar{e} \Phi^{\top} \mathcal{L} \Phi v=e^{\top} \bar{\kappa} \Upsilon \Phi^{\top} \mathcal{L} \Phi v$ and $\kappa^{\top} \Upsilon \bar{s} \Phi^{\perp \top} \mathcal{L} \Phi v=s^{\top} \bar{\kappa} \Upsilon \Phi^{\perp \top} \mathcal{L} \Phi v$, we obtain

$$
\begin{aligned}
\frac{d}{d t}\left[V \kappa^{\top} \Upsilon(t) \kappa\right] \leq- & \frac{\mu}{T} V|\kappa|^{2}+V \kappa^{\top} \dot{q}(t)^{2} \kappa \\
& +2 V \kappa^{\top} \Upsilon\left[\bar{e} \Phi^{\top} \mathcal{L} \Phi v+\bar{s} \Phi^{\perp \top} \mathcal{L} \Phi v\right] \\
\leq & -\frac{\mu}{T} V|\kappa|^{2}+V \kappa^{\top} \dot{q}(t)^{2} \kappa+2 V e^{\top} \bar{\kappa} \Upsilon \Phi^{\top} \mathcal{L} \Phi v \\
& +2 V s^{\top} \bar{\kappa} \Upsilon \Phi^{\perp \top} \mathcal{L} \Phi v .
\end{aligned}
$$

Then, after the triangle inequality, the following hold for any $\epsilon>0$ :

$$
\begin{aligned}
\frac{d}{d t}\left[V \kappa^{\top} \Upsilon(t) \kappa\right] \leq & -\frac{\mu}{T} V \kappa^{\top} \kappa+V \kappa^{\top} \dot{q}(t)^{2} \kappa \\
& +V\left[\frac{1}{\epsilon} e^{\top} \bar{\kappa} e+\epsilon v^{\top} \Phi^{\top} \mathcal{L} \Phi \Upsilon \bar{\kappa} \Phi^{\top} \mathcal{L} \Phi v\right] \\
& +V\left[\frac{1}{\epsilon} s^{\top} \bar{\kappa} s+\epsilon v^{\top} \Phi^{\perp \top} \mathcal{L} \Phi \Upsilon \bar{\kappa} \Phi^{\perp \top} \mathcal{L} \Phi v\right] \\
\leq & -\frac{\mu}{T} V \kappa^{\top} \kappa+V \kappa^{\top} \dot{q}(t)^{2} \kappa+V \frac{2}{\epsilon} \kappa^{\top} \kappa \\
& +V \epsilon v^{\top} \Phi^{\top} \mathcal{L} \Phi \Upsilon \kappa \Phi^{\top} \mathcal{L} \Phi v \\
& +V \epsilon v^{\top} \Phi^{\perp \top} \mathcal{L} \Phi \Upsilon \kappa \Phi^{\perp \top} \mathcal{L} \Phi v \\
\leq & -\left[\frac{\mu}{T}-\frac{2}{\epsilon}\right] V \kappa^{\top} \kappa+V \kappa^{\top} \dot{q}(t)^{2} \kappa \\
& +V^{2} \epsilon \lambda_{N}(L)\left[\left|\Upsilon \Phi^{\top} \mathcal{L} \Phi\right|_{\infty}^{2}+\left|\Upsilon \Phi^{\perp \top} \mathcal{L} \Phi\right|_{\infty}^{2}\right] \times \\
& \left|K_{d t} K_{p t}^{-1}\right| v^{\top} K_{d t} K_{p t}^{-1} v
\end{aligned}
$$

for which we also used the inequalities $s^{\top} \bar{\kappa} s+e^{\top} \bar{\kappa} e \leq 2 \kappa^{\top} \kappa$,

$$
\begin{array}{r}
v^{\top} \Phi^{T} \mathcal{L} \Phi \Upsilon \kappa \Phi^{T} \mathcal{L} \Phi v \leq \lambda_{N}(L) V\left|\Upsilon \Phi^{\top} \mathcal{L} \Phi\right|_{\infty}^{2} \times \\
\left|K_{d t}^{-1} K_{p t}\right| v^{\top} K_{d t} K_{p t}^{-1} v
\end{array}
$$

and

$$
\begin{array}{r}
v^{\top} \Phi^{\perp \top} \mathcal{L} \Phi \Upsilon \kappa \Phi^{\perp \top} \mathcal{L} \Phi v \leq \lambda_{N}(L) V\left|\Upsilon \Phi^{\perp \top} \mathcal{L} \Phi\right|_{\infty}^{2} \times \\
\left|K_{d t}^{-1} K_{p t}\right| v^{\top} K_{d t} K_{p t}^{-1} v .
\end{array}
$$

Next, using the inequality $V \kappa^{\top} \dot{q}(t)^{2} \kappa \leq V s^{\top} \dot{q}(t)^{2} \bar{\kappa} s+$ $b_{q}^{2} \lambda_{N}(L) V^{2}\left|K_{p t}^{-1}\right| e^{\top} K_{p t} e$, we obtain:

$$
\begin{aligned}
\frac{d}{d t}\left[V \kappa^{\top} \Upsilon(t) \kappa\right] \leq & -\left[\frac{\mu}{T}-\frac{2}{\epsilon}\right] V \kappa^{\top} \kappa+V s^{\top} \dot{q}(t)^{2} \bar{\kappa} s \\
& +b_{q}^{2} \lambda_{N}(L) V^{2}\left|K_{p t}^{-1}\right| e^{\top} K_{p t} e \\
& +V^{2} \epsilon \lambda_{N}(L)\left[\left|\Upsilon \Phi^{\top} \mathcal{L} \Phi\right|_{\infty}^{2}+\left|\Upsilon \Phi^{\perp \top} \mathcal{L} \Phi\right|_{\infty}^{2}\right] \times \\
& \left|K_{d t}^{-1} K_{p t}\right| v^{\top} K_{d t} K_{p t}^{-1} v .
\end{aligned}
$$

For the third term on the right-hand side of (37), we have

$$
\begin{aligned}
\frac{d}{d t}\left[\alpha(V) e^{\top} v\right] \leq & {\left[\frac{\partial \alpha}{\partial V} e^{\top} v\right] \dot{V}+\alpha(V)\left[v^{\top} \Phi^{\top} \mathcal{L} \Phi v+s^{\top} \dot{q} \bar{\kappa} v\right.} \\
& \left.-e^{\top} K_{d t} v-e^{\top} K_{p t} e-v^{\top} \bar{e}_{\omega} s-e^{\top} K_{p t} \Phi^{\top} \mathcal{A}\right] \\
\leq & -\alpha(V) e^{\top} K_{p t} e+\left[\frac{\partial \alpha}{\partial V} e^{\top} v\right] \dot{V} \\
& +\alpha(V) v^{\top}\left[\Phi^{\top} \mathcal{L} \Phi K_{d t}^{-1} K_{p t}\right] K_{d t} K_{p t}^{-1} v \\
& +\alpha(V) s^{\top} \dot{q} \bar{\kappa} v-\alpha(V) e^{\top} K_{p t} K_{d t} K_{p t}^{-1} v \\
& -\alpha(V) v^{\top} \bar{e}_{\omega} s-\alpha(V) e^{\top} K_{p t} \Phi^{\top} \mathcal{A}
\end{aligned}
$$




$$
\begin{aligned}
\leq & -\alpha(V) e^{\top} K_{p t} e+\left[\frac{\partial \alpha}{\partial V} e^{\top} v\right] \dot{V} \\
& +\alpha(V)\left|\Phi^{\top} \mathcal{L} \Phi\right|_{\infty}\left|K_{d t}^{-1} K_{p t}\right| v^{\top} K_{d t} K_{p t}^{-1} v \\
& +\frac{1}{\epsilon} s^{\top} \dot{q}^{2} \bar{\kappa}^{2} s+\frac{\epsilon}{4}\left|K_{p t} K_{d t}^{-1}\right| \alpha^{2}(V) v^{\top} K_{d t} K_{p t}^{-1} v \\
& +\frac{1}{\epsilon} \alpha(V) e^{\top} K_{p t} e+\frac{\epsilon}{4} \alpha(V)\left|K_{d t}\right| v^{\top} K_{d t} K_{p t}^{-1} v \\
& -\alpha(V) v^{\top} \bar{e}_{\omega} s-\alpha(V) e^{\top} K_{p t} \Phi^{\top} \mathcal{A}
\end{aligned}
$$

in which we used the inequalities

$$
\begin{aligned}
v^{\top} \Phi^{\top} \mathcal{L} \Phi v & \leq\left|\Phi^{\top} \mathcal{L} \Phi\right|_{\infty}\left|K_{d t}^{-1} K_{p t}\right| v^{\top} K_{d t} K_{p t}^{-1} v, \\
\alpha(V) s^{\top} \dot{q} \bar{\kappa} v & \leq \frac{1}{\epsilon} s^{\top} \dot{q}^{2} \bar{\kappa}^{2} s+\frac{\epsilon}{4}\left|K_{p t} K_{d t}^{-1}\right| \alpha^{2}(V) v^{\top} K_{d t} K_{p t}^{-1} v,
\end{aligned}
$$

and

$$
\alpha e^{\top} K_{d t} v \leq \frac{1}{\epsilon} \alpha(V) e^{\top} K_{p t} e+\frac{\epsilon}{4}\left|K_{d t}\right| \alpha(V) v^{\top} K_{d t} K_{p t}^{-1} v .
$$

Rearranging terms, we obtain

$$
\begin{aligned}
\frac{d}{d t}\left[\alpha(V) e^{\top} v\right] & \leq-\alpha(V) e^{\top} K_{p t} e\left[1-\frac{1}{\epsilon}\right] \\
& +\frac{\lambda_{N}(L)}{\epsilon} V s^{\top} \dot{q}^{2} \bar{\kappa} s-\alpha(V) v^{\top} \bar{e}_{\omega} s \\
& +\left[-\left[\frac{\partial \alpha}{\partial V} e^{\top} v\right]+\alpha(V)\left|\Phi^{\top} \mathcal{L} \Phi\right|_{\infty}\left|K_{d t}^{-1} K_{p t}\right|\right. \\
& \left.+\frac{\epsilon}{4}\left|K_{p t} K_{d t}^{-1}\right| \alpha^{2}(V)+\frac{\epsilon}{4} \alpha(V)\left|K_{d t}\right|\right] v^{\top} K_{d t} K_{p t}^{-1} v \\
& -\alpha(V) e^{\top} K_{p t} \Phi^{\top} \mathcal{A}+\frac{\partial \alpha}{\partial V} e^{\top} v Y\left(\dot{z}_{t}\right)
\end{aligned}
$$

Next, we evaluate the derivative of the fourth term on the righthand side of (37). We obtain

$$
\begin{aligned}
\frac{d}{d t}\left[-c_{1} V e^{\top} \dot{q} s\right] \leq & -e^{\top} \ddot{q} s V c_{1}-c_{1}\left[e^{\top} \dot{q} s\right] \dot{V} \\
& -c_{1} V\left[v^{\top} \Phi^{\top} \mathcal{L} \Phi \dot{q} s+s^{\top} \bar{\kappa} \dot{q}^{2} s-e^{\top} \dot{q}^{2} \bar{\kappa} e\right. \\
& \left.\quad+e^{\top} \dot{q} \Phi^{\perp \top} \mathcal{L} \Phi v-s^{\top} \bar{e}_{\omega} \dot{q} s+e^{\top} \dot{q} \bar{e}_{\omega} e\right] \\
\leq & -c_{1} V s^{\top} \dot{q}^{2} \bar{\kappa} s+\frac{\epsilon}{4} b_{q}^{2} c_{1}^{2} e^{\top} e+\frac{1}{\epsilon} V^{2} s^{\top} s \\
& +\left[c_{1} e^{\top} \dot{q} s\right] v^{\top} K_{d t} K_{p t}^{-1} v+\left[c_{1} e^{\top} \dot{q} s\right] Y\left(\dot{z}_{t}\right) \\
& +\frac{\epsilon}{4} c_{1}^{2}\left|K_{d t}^{-1} K_{p t}\right|\left|\dot{q}^{2}\left[\Phi^{\top} \mathcal{L} \Phi\right]^{2}\right|_{\infty} v^{\top} K_{d t} K_{p t}^{-1} v \\
& +\frac{1}{\epsilon} V^{2} s^{\top} s+b_{q}^{2} c_{1} \lambda_{N}(L) V^{2} e^{\top} e-c_{1} V e^{\top} \dot{q} \bar{e}_{\omega} e \\
& +\frac{1}{\epsilon} c_{1}\left|\dot{q}^{2}\left[\Phi^{\perp \top} \mathcal{L} \Phi\right]^{2}\right|_{\infty}\left|K_{d t}^{-1}\right| V e^{\top} K_{p t} e \\
& +\frac{\epsilon}{4} c_{1} V v^{\top} K_{d t} K_{p t}^{-1} v+c_{1} V s^{\top} \dot{q} \bar{e}_{\omega} s
\end{aligned}
$$

for which we used the inequalities:

$$
c_{1} V e^{\top} \ddot{q} s \leq \frac{\epsilon}{4} c_{1}^{2} b_{q}^{2} e^{\top} e+\frac{1}{\epsilon} V^{2} s^{\top} s,
$$

$c_{1} V v^{\top} \Phi^{\top} \mathcal{L} \Phi \dot{q} s \leq \frac{\epsilon}{4} c_{1}^{2}\left|\dot{q}^{2}\left[\Phi^{\top} \mathcal{L} \Phi\right]^{2}\right|_{\infty}\left|K_{d t}^{-1} K_{p t}\right| v^{\top} K_{d t} K_{p t}^{-1} v$

$$
+\frac{1}{\epsilon} V^{2} s^{\top} s
$$

and

$$
\begin{aligned}
c_{1} V e^{\top} \dot{q} \Phi^{\perp \top} L \Phi v \leq & \frac{1}{\epsilon} c_{1} V\left|\dot{q}^{2}\left[\Phi^{\perp \top} \mathcal{L} \Phi\right]^{2}\right|_{\infty}\left|K_{d t}^{-1}\right| e^{\top} K_{p t} e \\
& +\frac{\epsilon}{4} c_{1} V v^{\top} K_{d t} K_{p t}^{-1} v
\end{aligned}
$$

Therefore, using the inequality

$$
V^{2} s^{\top} s \leq V^{2} \lambda_{N}(L)\left|K_{d t}^{-1}\right| v^{\top} K_{p t}^{-1} K_{d t} v+\frac{4 N}{\lambda_{2}(L)} V \kappa^{\top} \kappa
$$

we obtain

$$
\begin{aligned}
\frac{d}{d t}[ & \left.-c_{1} V e^{\top} \dot{q} s\right] \leq-c_{1} V s^{\top} \dot{q}^{2} \bar{\kappa} s+\left[c_{1} e^{\top} \dot{q} s\right. \\
& \left.+\frac{\epsilon}{4} b_{q}^{2} c_{1}^{2}\left|K_{d t}^{-1} K_{p t}\right|\left|\left[\Phi^{\top} \mathcal{L} \Phi\right]^{2}\right|_{\infty}+\frac{\epsilon}{4} c_{1} V\right] v^{\top} K_{d t} K_{p t}^{-1} v \\
& +\left[b_{q}^{2} c_{1} \lambda_{N}(L)\left|K_{p t}^{-1}\right| V^{2}+\frac{1}{\epsilon} c_{1}\left|\dot{q}^{2}\left[\Phi^{\perp \top} \mathcal{L} \Phi\right]^{2}\right|_{\infty}\left|K_{d t}^{-1}\right| V\right. \\
& \left.+\frac{\epsilon}{4} b_{q}^{2} c_{1}^{2}\left|K_{p t}^{-1}\right|\right] e^{\top} K_{p t} e+\left[c_{1} e^{\top} \dot{q} s\right] Y\left(\dot{z}_{t}\right) \\
& +\frac{2}{\epsilon} V^{2} \lambda_{N}(L)\left|K_{d t}^{-1}\right| v^{\top} K_{p t}^{-1} K_{d t} v+\frac{8 N}{\lambda_{2}(L) \epsilon} V \kappa^{\top} \kappa \\
& +c_{1} V s^{\top} \dot{q} \bar{e}_{\omega} s-c_{1} V e^{\top} \dot{q} \bar{e}_{\omega} e .
\end{aligned}
$$

Putting the bounds (57), (58), (56), (55), and (54) together, we obtain

$$
\begin{aligned}
& \dot{W} \leq-\left[\gamma(V)-V^{2} \epsilon \lambda_{N}(L)\left[\left|\Upsilon \Phi^{\top} \mathcal{L} \Phi\right|_{\infty}^{2}\right.\right. \\
& \left.+\left|\Upsilon \Phi^{\perp \top} \mathcal{L} \Phi\right|_{\infty}^{2}\right]\left|K_{d t}^{-1} K_{p t}\right|+\left[\frac{\partial \alpha}{\partial V} e^{\top} v\right] \\
& -\alpha(V)\left|\Phi^{\top} \mathcal{L} \Phi\right|_{\infty}\left|K_{d t}^{-1} K_{p t}\right|-\frac{\epsilon}{4}\left|K_{p t}\right| \alpha(V)^{2}-\left[c_{1} e^{\top} \dot{q} s\right] \\
& -\frac{\epsilon}{4} \alpha(V)\left|K_{d t}\right|-\frac{\epsilon}{4} c_{1}^{2}\left|K_{d t}^{-1} K_{p t}\right|\left|\left[\Phi^{\top} \mathcal{L} \Phi\right]^{2} \dot{q}^{2}\right|_{\infty}-\frac{\epsilon}{4} c_{1} V \\
& \left.-\frac{2}{\epsilon} V^{2} \lambda_{N}(L)\left|K_{d t}^{-1}\right|\right] v^{\top} K_{d t} K_{p t}^{-1} v \\
& -\left[\alpha(V)\left[1-\frac{1}{\epsilon}\right]-b_{q}^{2} \lambda_{N}(L) V^{2}\left|K_{p t}^{-1}\right|\right. \\
& -b_{q}^{2} c_{1} \lambda_{N}(L)\left|K_{p t}^{-1}\right| V^{2}-\frac{1}{\epsilon} c_{1}\left|\dot{q}^{2}\left[\Phi^{\perp \top} \mathcal{L} \Phi\right]^{2}\right|_{\infty} \times \\
& \left.\left|K_{d t}^{-1}\right| V-\frac{\epsilon}{4} b_{q}^{2} c_{1}^{2}\left|K_{p t}^{-1}\right|\right] e^{\top} K_{p t} e+\left[c_{1} e^{\top} \dot{q} s\right] Y\left(\dot{z}_{t}\right) \\
& -\alpha(V) e^{\top} K_{p t} \Phi(\theta)^{\top} \mathcal{A}\left(\dot{z}_{t}\right)-\left[\frac{\partial \alpha}{\partial V} e^{\top} v\right] Y\left(\dot{z}_{t}\right) \\
& -\left[c_{1}-1-\frac{\lambda_{N}(L)}{\epsilon}\right] V s^{\top} \dot{q}^{2} \bar{\kappa} s-\left[\frac{\mu}{T}-\frac{2}{\epsilon}-\frac{8 n}{\lambda_{2}(L) \epsilon}\right] V|\kappa|^{2} \\
& -\alpha(V) v^{\top} \bar{e}_{\omega} s+c_{1} V s^{\top} \dot{q} \bar{e}_{\omega} s-c_{1} V e^{\top} \dot{q} \bar{e}_{\omega} e
\end{aligned}
$$

Then setting $\epsilon=\max \left\{2, \frac{2 T}{\mu}\left[2+\frac{8 N}{\lambda_{2}(L)}\right]\right\}$,

$$
\begin{aligned}
\gamma(V) \geq & 2 V^{2} \epsilon \lambda_{N}(L)\left[\left|\Upsilon \Phi^{\top} \mathcal{L} \Phi\right|_{\infty}^{2}+\left|\Upsilon \Phi^{\perp \top} \mathcal{L} \Phi\right|_{\infty}^{2}\right]\left|K_{d t}^{-1} K_{p t}\right| \\
& +\frac{\partial \alpha}{\partial V} V\left[\left|K_{p t}\right|+\lambda_{N}(L)\right]+\frac{\epsilon}{2} c_{1} V \\
& +2 \alpha(V)\left|\Phi^{\top} \mathcal{L} \Phi\right|_{\infty}\left|K_{d t}^{-1} K_{p t}\right|_{\infty}+\frac{\epsilon}{2}\left|K_{p t} K_{d t}^{-1}\right| \alpha^{2}(V) \\
& +\frac{\epsilon}{2} \alpha(V)\left|K_{d t}\right|+2 c_{1} b_{q} \lambda_{N}(L) V+\frac{4}{\epsilon} V^{2} \lambda_{N}(L)\left|K_{d t}^{-1}\right| \\
& +\frac{\epsilon}{2} c_{1}^{2}\left|K_{d t}^{-1} K_{p t}\right|\left|\dot{q}^{2}\left[\Phi^{\top} \mathcal{L} \Phi\right]^{2}\right|_{\infty} \\
\alpha(V) \geq & 4 b_{q}^{2} \lambda_{N}(L) V^{2}\left|K_{p t}^{-1}\right|+4 b_{q}^{2} c_{1} \lambda_{N}(L)\left|K_{p t}^{-1}\right| V^{2} \\
& +4 \frac{1}{\epsilon} c_{1}\left|\dot{q}^{2}\left[\Phi^{\perp \top} \mathcal{L} \Phi\right]^{2}\right|_{\infty}\left|K_{d t}^{-1}\right| V+4 \frac{\epsilon}{4} b_{q}^{2} c_{1}^{2}\left|K_{p t}^{-1}\right|
\end{aligned}
$$

and

$$
c_{1}=1+\frac{\lambda_{N}(L)}{\max \left\{2, \frac{2 T}{\mu}\left(1+\frac{2 N}{\lambda_{2}(L)}\right)\right\}},
$$

we obtain

$$
\begin{aligned}
\dot{W} \leq & -\frac{1}{2} \gamma(V) v^{\top} K_{d t} K_{p t}^{-1} v-\frac{1}{4} \alpha(V) e^{\top} K_{p t} e-\frac{\mu}{2 T} V|\kappa|^{2} \\
& -\alpha(V) v^{\top} \bar{e}_{\omega} s+c_{1} V s^{\top} \dot{q} \bar{e}_{\omega} s-\dot{q} c_{1} V e^{\top} \dot{q} \bar{e}_{\omega} e \\
& -\alpha(V) e^{\top} K_{p t} \Phi(\theta)^{\top} \mathcal{A}\left(\dot{z}_{t}\right) \\
& -\left[\gamma(V)-c_{1} e^{\top} \dot{q} s+\left[\frac{\partial \alpha}{\partial V} e^{\top} v\right]\right] Y\left(\dot{z}_{t}\right)
\end{aligned}
$$

